\title{
ADAPTIVE ESTIMATION USING PARALLEL PROCESSING
}

TECHNIQUES*

by

\author{
Peter K.S.Tamt \\ John B. Moore't \\ Technical Report FE7411 \\ ISBN $0 \quad 7259 \quad 0122$ \\ Revised August 1974
}

* Work supported by the Australian Research Grants Committee.

+ Department of Electrical Engineering, University of Newcastle

New South Wales, 2308

Australia 


\section{$\underline{\text { ABSTRACT }}$}

A parallel processing technique for adaptive estimation is investigated. In particular, for the case of unknown system or statistical parameters denoted by the vector $\theta$ belonging to a finite set $\left\{\theta_{1}, \theta_{2}, \ldots, \theta_{N}\right\}$, the maximum likelihood $\theta$ is determined and denoted $\hat{\theta}$, and the minimum mean square error state estimate conditioned on this $\hat{\theta}$, namely $\hat{x}(t \mid t, \hat{\theta})$ is taken to be the state estimate. Using this approach new estimators are derived which require less computational effort and have less limitations than previous adaptive estimators using parallel processing techniques described in the iiterature. Results for the case of time-varying unknown parameters are also derived.

An example is included of state estimation for a known signal model but with unknown noise statistics. The filter banks are constrained to be time-invariant and so only appropimate maximum likelihood parameter estimation is achieved. 


\section{Introduction}

The Kalman-Bucy filter $[1,2]$ for the estimation of the states of a linear dynamical system requires an exact knowledge of the system parameters and noise covariances. We consider the adaptive estimation problem of estimating the states when the dynamical and/or statistical model is specified up to a set of unknown parameters, denoted by the vector $\theta$.

Parallel processing techniques have been applied by a number of authors [3-5] to the adaptive estimation problem and in fact adaptive estimators requiring one hundred or so kalman filters can be implemented using mini-computers. In essence, tire standard bavesian approach to the adaptive estimation problem is as follows [5]. Assuning that the unknown parameter vector $\theta$ is discrete or suitablv quantized to a finite number of grid points $\{\ldots, \ldots$, , with known or assumed a priari probalility for each " $i$, the conditional mean estimator includes a bank of it kalman filters where the $i^{\text {th }}$ filter is a standard falman filter designed on the assumption that $=n_{j}$. The filter bank is driven by the noisy signal measurements. The conditional mean state estimate is given by a weignted sum of the states of the kalman filters. The weighting coefficient of the state of the $i^{\text {th }}$ kalman filter is the a posteriori probability of $\theta_{i}$, which can be updated recursively using the noisy signa measurements and the state of the $i^{\text {th }}$ kalman filter.

Unfortunately, for systems with continuous-time meascirements, the above Eayesian approach has the drawback that, first, the measurement noise covariance $P$ has to we known, and second, the unknown parameter vector $\theta$ has to be time-invariant. 
For the special case of systems with discrete measurements, parallel processing estimation techniques have been developed when the measurement noise covariance is unknown, at the expense of either complexity or loss of optimality [4], [6]. In the practically important direction of reducing the complexity of the adaptive estimator, A1spach and Abiri $[6,7]$ obtain time-invariant state estimators for time-invariant systems with unknown noise covariances by considering a grid of possible time-invariant Kalman gains directly ratier than a more involved grid of possiule noise covariances in the unknown parameter space. The results can be extended to cases when tine noise covariances are time-varying quantities [8]. However, for the estimator of [6] to be close to optimal, it is required that the kalman filters have reacned steady-state and that the number of measurements received be large. Thus, during the transient periods, the estimator operates suboptimally.

Some parallel processing estimation techniques are also available for discrete-time systems when 0 is time-varying. Using a combination of digital and analogue techniques, optimal adaptive estimators for the case when the unknown parameter is a scalar Markov sequence of known statistics have been developed [9]. By approximating the a posteriori density of the state vector with a Gaussian probability density, Ackerson and Fu [10] derived a suboptimal estimator for the particular time-varying unknown parameter case in which the input noise or the measurement noise comes from a group of white Gaussian noise sources, which act one at a time, with the transition from one noise source to another being described by a discrete Markov transition matrix.

It is clear from the above survey of existing adaptive estimators using parallel processing techniques that it would be 
worthwhile to investigate any adaptive estimation approacn using parallel processing teciniques suitable for botin continuous-time and discrete-time problems, and which can handle unknown time-varying parameters and unknown measurement noise parameters with but a small addition in complexity over the case of time-invariant unknown parameters and known measurement noise covariance. In this paper, such an approach is investigated. This approach can be briefly described as follows.

An unknown parameter vector $\theta$ is defined in such a way that there is one and only one Kalman filter corresponding to a particular arid point $\theta_{i}$ in the 0 space. The states of the kalman filters are denoted $\hat{x}\left(t \mid t, 0_{i}\right)$. By comparing the relative magnitudes of the likelihood functions of tile $\theta_{i}$ 's evaluated using the measurement data and $\hat{x}\left(t \mid t,{ }_{j}\right)$ for all $i$, the most likely ${ }_{i}$ at time $t$, denoted $\hat{\theta}(t)$, is deternined. The state $\hat{x}[t \mid t, \hat{\theta}(t)]$ is taken as the estimate of the signal model state $x(t)$. Note that since the likelihood functions of the unknown parameters are used instead of their exact a posteriori probabilities, it is not necessary to assign a priori probabilities to the different $0_{i}$ 's

The advantages of using the approach just outlined can be sumnarised as follows. First, for continuous-time systems, in contrast to the standard Bayesian approach, our approach does not require that the measurement noise covariance be known exactly. Second, for the discrete-time case, our estimators require less computational effort than alternative known estimators using parallel processing techniques. Third, for both the continuous-time case and the discrete-time case, the various results can be extended in a simple manner to give useful adaptive estimators for the case of time-varying $\theta$. 
The above advantages are of course useless unless the estimators using the above approach perform satisfactorily. Monte Carlo simulations show that they work very well in minimizing the mean square estimation error when compared to the more complex adaptive estimator for discretetime problems described in [8].

An outline of the subsequent sections of this paper is as follows. In section II, we present the discrete-time results for time-invariant unknown parameters. In section III, the results of section II are extended to the case of time-varying unknown parameters. In section IV, the results of sections II, III are extended to systems with continuoustime measurements. 


\section{DISCRETE-TIME RESULTS FOR TIML IHVRRIAMT UNKKIO'HI PARAMI TLPS}

In this section, we first review appropriate discrete-tine optinal adaptive estinator results from [3]-[5] and then we apply these results to achieve an alternative adaptive estindor which in most nuplications is simpler and thus more attractive. A simulation example is miven to delionstrate the perforiance characteristics of the altemative estinator. Consider the system

$$
\begin{aligned}
& x\left(t_{k+1}\right)=\left(t_{k+1}, t_{k} \mid i_{i}\right) x\left(t_{k}\right)+G\left(t_{k}\right) u\left(t_{k}\right) \\
& y\left(t_{k+1}\right)=11\left(t_{k+1} \mid+x\left(t_{k+1}\right)\right. \\
& z\left(t_{k+1}\right)=y\left(t_{k+1}\right)+v\left(t_{k+1}\right)
\end{aligned}
$$

winere $u(\cdot), v(\cdot)$ are independent zero-mean Gaussian white nuise sequences with covariance matrices $I$ and $R$ respectively. The positive definite matrix $R$ will be assumed known in some instances, and not known in others. The initial state $x\left(t_{0}\right)$ is a Gaussian random vector with mean $x_{0}(i \psi)$ and variance $P\left(t_{0} \mid t_{0}, \psi\right)$ and is independent of $u(\cdot)$ and $v(\cdot)$. The entities $\left.t_{k+1}, t_{k} \mid \psi\right), G\left(t_{k} \mid w_{1}\right), H\left(t_{k+1} \mid \cdot\right), \times(\therefore), P\left(t_{0} \mid t_{0},\right)$ are completely specified by the parameter vector , i.e. they are known if $\psi$ is known, possibly unknown if $;$ is not known. This formulation effectively allows an unknown input noise covariance (a) though we have assumed $\left.E\left\{u\left(t_{k}\right) u \cdot\left(t_{k}\right)\right\}=I\right)$ througin the intervention of $G\left(t_{k} \mid \ldots\right)$ in (1).

If $\psi$ and $R$ are specified, the conditional mean state estinate $\hat{x}\left(t_{k}, t_{k}, \psi, R\right)$ is of course given from the familiar kalman filter equations: 


$$
\begin{aligned}
& \hat{x}\left(t_{k+1} \mid t_{k+1}, \psi, R\right)=\hat{x}\left(t_{k+1} \mid t_{k}, \psi, R\right)+K\left(t_{k+1} \mid \psi, R\right) \tilde{z}\left(t_{k+1} \mid \psi, R\right) \\
& \hat{x}\left(t_{k+1} \mid t_{k}, \psi, R\right)=\phi\left(t_{k+1} \mid t_{k}, \psi\right) \hat{x}\left(t_{k} \mid t_{k}, \psi, R\right), \hat{x}\left(t_{0} \mid t_{0}, \psi, R\right)=\hat{x}_{0}(\psi)(5) \\
& \tilde{z}\left(t_{k+1} \mid \psi, R\right)=z\left(t_{k+1}\right)-H\left(t_{k+1} \mid \psi\right) \hat{x}\left(t_{k+1} \mid t_{k}, \psi, R\right) \\
& K\left(t_{k+1} \mid \psi, R\right)=P\left(t_{k+1} \mid t_{k}, \psi, R\right) H^{\prime}\left(t_{k+1} \mid \psi, R\right) P_{z}^{-1}\left(t_{k+1} \mid t_{k}, \psi, R\right) \\
& P_{z}\left(t_{k+1} \mid t_{k}, \psi, R\right)=H\left(t_{k+1} \mid \psi\right) P\left(t_{k+1} \mid t_{k}, \psi, R\right) H^{\prime}\left(t_{k+1} \mid \psi\right)+R \\
& P\left(t_{k+1} \mid t_{k}, \psi, R\right)=\phi\left(t_{k+1}, t_{k} \mid \psi\right) P\left(t_{k} \mid t_{k}, \psi, R\right) \phi^{\prime}\left(t_{k+1}, t_{k} \mid \psi\right)+G\left(t_{k} \mid \psi\right) G^{-}\left(t_{k} \mid \psi\right) \\
& P\left(t_{k+1} \mid t_{k+1}, \psi, R\right)=P\left(t_{k+1} \mid t_{k}, \psi, R\right)-K\left(t_{k+1} \mid \psi, R\right) P_{z}\left(t_{k+1} \mid t_{k}, \psi, R\right) K^{-}\left(t_{k+1} \mid \psi, R\right)
\end{aligned}
$$

ilext we consider that $R$ is known but $\psi$ is unknown. Assuming that $\psi$, the space of admissible values of $\psi$, is discrete or suitably quantized +a finite number of grid points $\left\{\psi_{1}, \ldots, \psi_{p}\right\}$, with known or assumed a priori probability $p\left(\psi_{i} \mid t_{0}, R\right)$ for eacis $\psi_{i}$, the conditional mean state estimate $\hat{x}\left(t_{k}\left|t_{k}, R\right\rangle\right.$ is given by [3]-[5]:

$$
\begin{aligned}
& \hat{x}\left(t_{k} \mid t_{k}, R\right)=\sum_{i=1}^{P} \hat{x}\left(t_{k} \mid t_{k}, \psi_{i}, R\right) p\left(\psi_{j} \mid t_{k}, R\right) \\
& p\left(\psi_{j} \mid t_{k}, R\right)=c\left|P_{z}\left(t_{k} \mid t_{k-1}, \psi_{j}, R\right)\right|^{-\frac{2}{2}} \exp \left\{-\left.\frac{z}{z}\left|\tilde{z}\left(t_{k} \mid \psi_{j}, R\right)\right|\right|_{p_{z}^{-1}} ^{2}\left(t_{k} \mid t_{k-1}, \psi_{j}, R\right)\right\} \\
& p\left(\psi_{i} \mid t_{k-1}, R\right)
\end{aligned}
$$

where $c$ is a normalizing constant independent of $\psi_{i}$ so that $\sum_{i=1}^{p} p\left(\psi_{i} \mid t_{k}, R\right)=1$.

Now consider the more general case where $R$ is unknown with a continuous range $R$ of admissible values. An obvious approach for extendina the above results is to first approximate $R$ with a suitable finite set of quantized points $\left\{R_{1}, \ldots, R_{M}\right\}$. 
Then with known or assumed a priori probability $p\left(v_{i}, R_{j} \mid t_{0}\right)$ for each $\left({ }_{i}, R_{j}\right)$, the conditional mean state estimate would be given from [4]

$$
\begin{gathered}
x\left(t_{k} \mid t_{k}\right)=\sum_{i=1}^{p} \sum_{j=1}^{M} \hat{x}\left(t_{k} \mid t_{k}, \psi, R_{j}\right) p\left(\psi_{j}, R_{j} \mid t_{k}\right) \\
p\left(\psi_{j}, R_{j} \mid t_{k}\right)=\left.c^{\prime} P_{z}\left(t_{k} \mid t_{k-1}, \psi_{j}, R_{j}\right)\right|^{-\frac{2}{2}} \exp \left\{-\left.||\left|z\left(t_{k} \mid \psi_{j}, R_{j}\right)\right|\right|^{2} P_{z}^{-1}\left(t_{k} \mid t_{k-1}, \psi_{j}, R_{j}\right)\right\} \\
p\left(\psi_{j}, R_{j} \mid t_{k-1}\right)
\end{gathered}
$$

where $c^{\prime}$ is a normalizing constant independent of ${ }_{i}$ and $R_{j}$.

To implement equation (13), $P \times$ halman filters are required compared to the requirement of $P$ Kalman filters for the implementation of equation (11). Tinis represents a large increase in the number of parallel processing units when $R$ is unknown.

As a first step towards reducing the filter complexity, we replace the above discretization of the Cartesian product $y \times R$ with the discretization of an alternative set which we now describe. Through equations (7) - (10), the product space $\psi \times R$ defines a (continuous) space 0 of hypotheses of possible Kalman filter configurations, specified by the quadruples $\left\{K(\cdot \mid \psi, R), \phi(\cdot \mid \psi), \hat{x}_{0}(\psi), H(\cdot \mid \psi)\right\}$. (In many cases, the various time-varying Kalman gains have to be approximated by time-invariant gains to simplify calculations). For each $\theta$ in $\theta$, we have the following equations in lieu of equations $(4)-(6)$.

$$
\begin{aligned}
& \hat{x}\left(t_{k+1} \mid t_{k+1}, \theta\right)=\hat{x}\left(t_{k+1} \mid t_{k}, \theta\right)+k\left(t_{k+1} \mid \theta\right) \tilde{z}\left(t_{k+1} \mid \theta\right) \\
& \hat{x}\left(t_{k+1} \mid t_{k}, \theta\right)=\phi\left(t_{k+1}, t_{k} \mid \theta\right) \hat{x}\left(t_{k} \mid t_{k}, \theta\right) \quad \hat{x}\left(t_{0} \mid t_{0}, \theta\right)=\hat{x}_{0}(\theta) \\
& \tilde{z}\left(t_{k+1} \mid \theta\right)=z\left(t_{k+1}\right)-H\left(t_{k+1} \mid \theta\right) \hat{x}\left(t_{k+1} \mid t_{k}, \theta\right)
\end{aligned}
$$


ilote that while equations (4)-(6) can be re-written in terms of 0 as above, it is not possible to do so with equations (7)-(10). Thus the mapping $R+0$ incurs some loss of information as the specification of alone determines only the Kalman filter configuration but not the error covariance of the state estimate associated with that lialman filter.

It turns out that if ; is to be approximated by a set of i! grid points $\left\{i_{1}, \ldots, g_{1}\right\}^{\prime}$ then in many cases $N$ can be much smaller than $P \times M$. As an illustration of the above statement, we point out that in $[f]$, a numerical example for state estimation of a linear time-invariant system with unknown noise covariances is given whereby 2500 grid points in the space of unknown noise covariances can be adequately replaced by 100 grid points in the space of possible steady-state Kalman gains.

Using the set of grid points $\left\{0_{1}, \ldots, N\right\}$, the conditional mean state estimate is now given from

$$
\hat{x}\left(t_{k} \mid t_{k}\right)=\sum_{i=1}^{N} \hat{x}\left(t_{k} \mid t_{k}, \theta_{i}\right) p\left(\hat{\theta}_{i} \mid t_{k}\right)
$$

where $p\left(u_{j} \mid t_{k}\right)$ is the a posteriori probability that $\theta=u_{i}$.

A crude approach for calculating $p\left(0_{j} \mid t_{k}\right)$ is to first observe that the joint a posteriori probability that $\theta=\theta_{i}$ and $R=R_{j}$ can be updated recursively using the following equation

$$
\begin{gathered}
p\left(\theta_{i}, R_{j} \mid t_{k}\right)=c \cdots\left|P_{z}\left(t_{k} \mid t_{k-1}, \theta_{j}, R_{j}\right)\right|^{-2} \exp \left\{-\frac{z_{z}}{2}\left(t_{k} \mid \theta_{j}\right)||^{2} p_{z}^{-1}\left(t_{k} \mid t_{k-1}, \theta_{j}, R_{j}\right)\right\} \\
p\left(\theta_{i}, R_{j} \mid t_{k-1}\right)
\end{gathered}
$$

where $C^{-1}$ is a normalizing constant independent of ${ }_{i}, R_{j}$; and $P_{z}\left(t_{k} \mid t_{k-1}, \theta_{i}, R_{j}\right)=E\left\{\tilde{z}\left(t_{k} \mid \theta_{j}\right) z^{-}\left(t_{k} \mid \theta_{j}\right) \mid R_{j}\right\}$ is related to $\left[K\left(t_{k} \mid \theta_{i}\right), R_{j}\right]$ through the following relation (c.f. equations $(7),(8)$ ), which holds when 
$p\left(\cdot_{i}, R_{j} \mid t_{0}\right) \neq 0$

$$
P_{z}\left(t_{k} \mid t_{k-1}, i_{i}, R_{j}\right)=\left[I-H\left(t_{k} i{ }_{i}\right) K\left(t_{k} i j\right)\right]^{-} \cdot r_{i}
$$

Then by approximating the Cartesian product space . I? with a set of grid points $\left(a_{i}, R_{j}\right) i=1, \ldots, i l j j=1, \ldots, i \cdot$ and applying the theorem of total probability, we can express $p\left(\cdots_{i}, t_{k}\right)$ ds a maroinal probability, viz,

$$
\mu\left(\because_{j} \mid t_{k}\right)=\sum_{j=1}^{\| 1} p\left(i_{j}, R_{j} \mid t_{k}\right)
$$

This approach of using equations (16)-(18) however requires considerable on-line calculations as we have to store and update lit separate quantities to obtain a single $p\left({ }_{i} \mid t_{k}\right)$. Moreover, a lot of off-line calculations may be required to obtain the a priori probatilities $p\left({ }_{i}, R_{j} \mid t_{0}\right)$.

An alternative approach is to ind an approximate expression for $p\left(v_{j} \mid t_{k}\right)$. For example, using the techniques described in [6], it can be shown that for scalar measurements and assuming that for each " i the range of admissible values of $P_{z}\left(t_{k} \mid t_{k-1}, r_{j}, R\right)$ is the interval $(0, \cdot)$, then we have the following approximate expression

$$
\left.p\left(o_{i} \mid t_{k}\right)=c \int_{-k}^{1} \sum_{j=1}^{k} z^{2}\left(t_{j} \mid u_{i}\right)\right]^{\frac{2-k}{2}}
$$

where $c$ is a normalizing constant independent of $i$ and $k$ is a large even number (greater than about 1000).

We now propose a simple estimation scheme to yieid a suboptimal minimum mean square error estimate for the case when $R$ is unknown with a 
continuous range of admissible values. Our approach is based on the observation (shown below) that under a simplifying assumption on the range of admissible values of $R$, then given the measurements up to time $t_{k}$, it is relatively easy to find $\left[\hat{\theta}\left(t_{k}\right), \hat{R}\left(t_{k}\right)\right]$, which we shall denote simply as $(\hat{\theta}, \hat{R})$, that maximizes the likelihood function $p\left(Z_{k} \mid \theta, R\right)$ of $(\theta, R)$, where $Z_{k}=\left\{z\left(t_{1}\right), \ldots, z\left(t_{k}\right)\right\}$. Using the estimate $\hat{\theta}$ so obtained, our proposed simple estimator is one yielding the estimates $\hat{x}\left(t_{k} \mid t_{k}, \hat{\theta}\right)$. (Note that $\hat{R}$ is not required other than as a step to find $\hat{\theta})$.

Using the fact that $E\left\{\tilde{z}\left(t_{j} \mid \theta_{i}\right) \tilde{z}{ }^{\prime}\left(t_{j} \mid \theta_{i}\right) \mid R\right\}=\left[I-H\left(t_{j} \mid \theta_{i}\right) K\left(t_{j} \mid \theta_{i}\right)\right]^{-2} R$, the likelihood function $\mathrm{p}\left(Z_{k} \mid \theta_{j}, R\right)$, which is the conditional probability density of $Z_{k}$ as a function of $\theta_{i}$ and $R$, can be established as

$$
\begin{aligned}
P\left(Z_{k} \mid \theta_{i}, R\right)= & \underset{j=1}{k}\left\{| 2 \pi R | ^ { - \frac { 1 } { 2 } } | I - H ( t _ { j } | \theta _ { i } ) K ( t _ { j } | \phi _ { j } ) | ^ { 2 } \operatorname { e x p } \left\{-\sum_{j=1}^{k}||\right.\right. \\
& \left.\tilde{z}\left(t_{j} \mid \theta_{j}\right)||^{2} R^{-1}\left[I-H\left(t_{j} \mid \theta_{j}\right) K\left(t_{j} \mid 0_{j}\right)\right]\right\}
\end{aligned}
$$

How we make the simplifying assumption (quite similar to that made in [6] when the measurements are scalars) that for each ${ }_{j}, R$ can take any value provided it is a positive definite symmetric matrix. For each $1={ }^{\theta}$ let $\hat{R}\left(\theta_{i}\right)$ denote the value of $R$ which maximizes $p\left(z_{k} \mid \theta_{j}, R\right)$. If $\hat{R}\left(O_{i}\right)$ exists, it can be obtained by setting $\frac{\partial p\left(Z_{k} \mid \theta_{i}, R\right)}{\partial R^{-}}=0$, which qives
for $k>1$,

$$
\hat{R}\left(0_{i}\right)=\frac{3}{2}(s+i)
$$

where

$$
\Omega=\frac{1}{k} \sum_{j=1}^{k} \tilde{z}\left(t_{j} \mid \theta_{i}\right) \tilde{z}^{-}\left(t_{j} \mid \theta_{i}\right)\left[I-H\left(t_{j} \mid \theta_{i}\right) k\left(t_{j} \mid \theta_{j}\right)\right]^{\prime}
$$


Substituting the above value of $P_{(}\left({ }_{i}\right)$ into the expression for $p\left(\underline{z}_{k} \mid e_{i}, R\right)$ gives

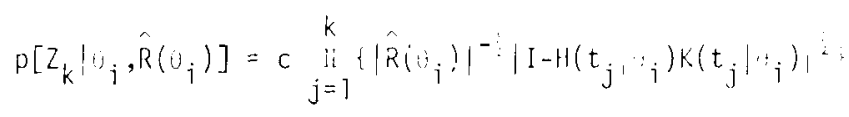

where $c$ is a constant independent of $\left[\because{ }_{j}, R\left(v_{j}\right)\right]$.

Equations (21) and (22), though useful in a number of cases, have to be used with caution because they apply only when $R\left(u_{i}\right)$ so obtained is positive definite. (Hence they are not applicabie for $k=1$ ). In orler to ensure a positive definite $\hat{R}\left(0_{i}\right)$, we need further assumption on the range of $R$ for each $=u_{i}$. Let us assume that for $\cdots={ }^{\prime}{ }_{i}, R$ is of tire form $R=a_{i} R_{i}$ where $R_{i}$ is a known posicive definite matrix and $i$ is an unknown scalar which can take any value in $(0, \cdots$. Then setting $\frac{\partial p\left(z_{k} \mid O_{i}, R\right)}{\partial i}=0$ gives

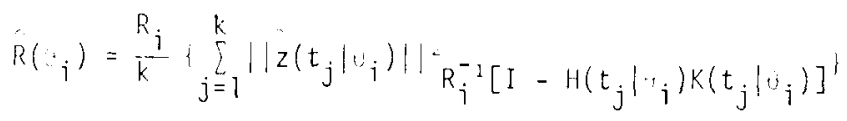

This equation (24) gives a positive definite $R\left(\theta_{j}\right)$ for all $k=1,2,3, \ldots$ Substituting this $\left.\hat{R}_{\left({ }_{i}\right.}\right)$ into the expression for $p\left(z_{k} \mid a_{j}, R\right)$ again gives equation (23).

iote that $\left.\hat{R}_{\left(O_{j}\right.}\right)$ and $\left.p\left[Z_{k} \mid \hat{\theta}_{i}, \hat{R}_{\left({ }_{j}\right.}\right)\right]$ can be updated recursiveiy, and that $\left.\max _{i}\{i)\left[z_{k} \mid \sigma_{i}, \hat{R}\left(v_{i}\right)\right]\right\}$ is in fact the global maximum of $p\left(z_{k} \mid \cdots_{i}, R\right)$ with respect to $i$ and $R$.

Dur proposed estimator consists of a bank of "I kalman filters. The conditional innovation processes of the lialman filters are used to update recursively $p\left[Z_{k} \mid \theta_{j}, \hat{R}\left(\theta_{j}\right)\right]$, by means of equation (23). Then $\hat{\theta}$ is chosen to maximize $p\left[Z_{k} \mid \theta_{i}, \hat{R}\left(\theta_{j}\right)\right]$, and $\hat{x}\left(t_{k} \mid t_{k}, \theta\right)$ is chosen as the state estimate. 
Such an estimator is sketched diagrammatically in Figure 1.

Under certain circumstances, the computations can be further simplified. For example, if the measurements are scalars, then as maybe seen from equation (23), the problem of maximizing $p\left(Z_{k} \mid \theta, R\right)$ with respect to $(\theta, R)$ reduces to the simpler problem of minimizing $\left\{\sum_{j=1}^{k} z^{2}\left(t_{j} \mid{ }_{i}\right) \mid i=1, \ldots N\right\}$

\section{Application to state estimators in unknown noise environment.}

It is interesting to compare the performance of our proposed estimator with the estimator proposed by Alspach and Abiri in [6] for state estimation of a linear time-invariant system when both the plant driving noise covariance $Q$ and the measurement noise covariance $R$ are unknown. In [6], to reduce the complexity of the filter structure, the $[Q, R]$ space is mapped to the space of Kalman gains (assumed to be time invariant). The simplifying assumption that tire Kalman gains are time invariant leads to sub-optimal performance during the transient period but is still a very useful assumption to make. In order to provide conditions for comparison purposes we will also make this assumption for our proposed estimator. Thus we consider the grid $\left\{0_{1}, \ldots, 0_{N}\right\}$ to be the arid of possible time-invariant (sub-optimal) Kalman gains.

He now consider the state estimation of a first-order system where the noise covariances are unknown. The system equations are

$$
\begin{aligned}
x\left(t_{k+1}\right) & =0.8 x\left(t_{k}\right)+G u\left(t_{k}\right) \\
z\left(t_{k}\right) & =x\left(t_{k}\right)+J v\left(t_{k}\right)
\end{aligned}
$$

where $u(\cdot), v(\cdot)$ are zero-mean independent white Gaussian sequences with unity covariances and $G, J$ are 'unknown' quantities.

The unknown kalman gain for the above system can take a value between 0 and 1. Ue consider the following simple Kalman gain space consisting of 
$N$ grid points $\left\{\theta_{1}, \ldots, \theta_{1}\right\}$ as the unknown parameter space. The ith kalman filter (that is the filter conditioned on $:=u_{j}$ ) has kalman gain $K\left(0_{i}\right)=\theta_{i}$ where $\theta_{i}=(i-.5) /_{i}$.

For the simulation results, the signal generating system is first run until steady-state can be assumed. Then measurements $z(k)$ are processed for estimation of $x\left(t_{k}\right)$ for $k=0,1, \ldots, 21$. Typical performances (the meansquare-error of the state estimates at each $t_{k}$ ) are shown in figures 2,3 using 300 sample paths and 10 grid points in the kalman gain space. The results show that our estimator actually performs slightly better than that of $[6]$.

To conclude this section we comment that not only does our scheme give better performance than that of $[0]$, at least for the somewhat random examples chosen, but it is certainly considerably less complicated to implement. 


\section{DISCRETE-TIME RESULTS FOR TIME-VARYIHG UIIKNOHN PARAMETERS}

In this section, we show that the estination scheme described in the last section can be extended in a simple but suboptimal manner to handle unknown time-varying (but not slowly varyina) parameters and unknown measurement noise parameters. The addition in complexity, which involves feedrack from the estimator output to the individual ralman filters, is very small relative to that required for an optimal minimum square error estimation scheme.

$$
\begin{aligned}
& \text { For our problem, equations (4)' - (6)' generalize to } \\
& \left.\hat{x}\left[t_{k+1} \mid t_{k+1}, O\left(t_{k+1}\right)\right]=\hat{x}\left[t_{k+1} \mid t_{k}, O\left(t_{k}\right)\right]+k\left[t_{k+1} \rho\left(t_{k+1}\right)\right] \tilde{z}\left[t_{k+1}\right)\left(t_{k}\right)\right] \\
& \hat{x}\left[t_{k+1} \mid t_{k}, \Theta\left(t_{k}\right)\right]=\psi\left[t_{k+1}, t_{k} \mid \theta\left(t_{k}\right)\right] \hat{x}\left[t_{k} \mid t_{k}, \Theta\left(t_{k}\right)\right], \quad \hat{x}\left[t_{0} \mid t_{0}, i\left(t_{0}\right)\right] \\
& =\hat{x}\left[\theta\left(t_{0}\right)\right] \\
& \left.\hat{z}\left[{ }_{k+1}^{t} 10\left(t_{k+1}\right)\right]=z\left(t_{k+1}\right)-1 i\left[t_{k+1}\right)\left(t_{k+1}\right)\right] \hat{x}\left[t_{k+1} \mid t_{k}, \theta\left(t_{k}\right)\right]
\end{aligned}
$$

where $\theta\left(t_{k}\right)=\left\{\theta\left(t_{0}\right), \ldots, \theta\left(t_{k}\right)\right.$.

Here we have to assume that for $\theta=\theta_{i}$ the measurement noise covariance $R\left(t_{k}\right)$ for $v\left(t_{k}\right)$ is of the form $R\left(t_{k}\right)=\alpha\left(t_{k}\right) R_{j}\left(t_{k}\right)$ where $\alpha\left(t_{k}\right)$ is an unknown scalar and $R_{j}\left(t_{k}\right)$ is a known matrix. We assume that $a\left(t_{k}\right), \theta\left(t_{k}\right)$, $\theta\left(t_{k-1}\right)$ are independent of eacil other and that $a\left(t_{k}\right)$ can take any value in $(0, \infty)$. The extimator structure that we are going to propose can be slightiy modified to include the case where there is a particular range of admissible values of $\theta\left(t_{k-1}\right)$ for each $\theta\left(t_{k}\right)$. However, simulation results so far indicate that very little improvement in performance can be gained by imposing such restrictions. 


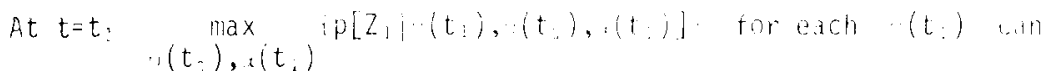
be found in two stages as follows. Firet, motr thit we can express

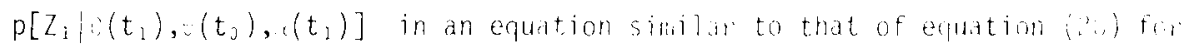
$p\left[z_{k} \mid \theta_{j}, R\right]$. Therefore, for each of the gridpoints $\left[i_{i}(t), j(t)\right]$

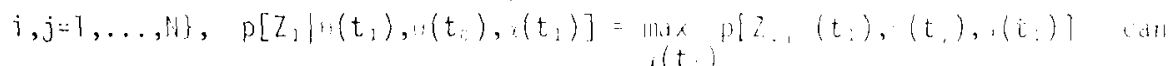
be found via a pair of equations similar to that of equations (23) and (a.7). Second, $\hat{o}\left(t_{0}\right)$ is chosen such that $p\left[z_{i} \mid \cdots\left(t_{i}\right),(t),\left(t_{i}\right)\right]$ $=\max _{0(t)}\left\{p\left[z_{1} \mid \theta\left(t_{1}\right), \theta\left(t_{0}\right), \hat{w}\left(t_{1}\right)\right]\right\}$ where $(t .1)$ can in qeneral be different for different $u\left(t_{1}\right)$. Having thus found $\because\left(t_{2}\right)$ for each $\cdot\left(t_{1}\right)$, the one step ahead prediction state estimate given by to bian filter conditioned on each $:\left(t_{1}\right)$ is then reset to $x\left[t_{i} \mid t, \ldots(t)\right]$, where again $\left.x\left[t_{1}, t, t_{i}\right)\right]$ can be different for different $:\left(t_{i}\right)$.

To recapitulate, based on the 'Mdximum litelihood' approach as describel in the last section and under some simplifying assumetions, we ruject, for each $\theta\left(t_{1}\right)$ at $t=t_{1}$, all the combinations of $\left.\left[\cdot(t), t_{1}\right)\right]$ except the peir $\left[\hat{\theta}\left(t_{0}\right), \hat{w}\left(t_{1}\right)\right]$ which maximizes the likelinood function $p\left[z_{1} \mid \cdots\left(t_{1}\right),(t),\left(t_{1}\right)\right]$.

In general, at $t=t_{k}, \underset{g\left(t_{k-1}\right),\left(t_{k}\right)}{\max } P\left[z_{k},\left(t_{1}\right),\left(t_{k-1}\right),\left(t_{k-2}\right),\left(t_{k}\right), A\left(t_{k-1}\right)\right.$ for each $u\left(t_{k}\right)$ can be found in two stacjes simjiar to those describrd above for $t=t_{1}$. Here $\hat{\theta}\left(t_{k-2}\right)=\left(\cdots\left(t_{1}\right), \cdots\left(t_{i}\right), \ldots, \cdots\left(t_{k-2}\right)\right.$. and $\hat{A}\left(t_{k-1}\right)=\left\{x\left(t_{1}\right), \ldots,\left(t_{k-1}\right)\right\}$ and these two seguences can in general be different for different $i\left(t_{k-1}\right)$. Having thus obtained $\|\left(t_{k-1}\right)$ for each $\theta\left(t_{k}\right)$, the one step ahead prediction state fistimite given by the kalman filter conditioned on each $\theta\left(t_{k}\right)$ is then reset to $x_{-}\left[t_{k} \mid t_{k-1},\left(t_{k-1}\right)\right]$. 
Resetting the states of the Kaiman filters as described above allows lis to limit the number of Kalman filters to a finite number in although the number of grid points for $o\left(t_{k}\right)$ grows exponentially with $k$.

As an illustration of the above procedure, we shall describe the derivation of the various guantities in more details for the case of scalar measurements (i.e. $R\left(t_{k}\right)=a_{a}\left(t_{k}\right)$ ) and $H\left[t_{k} \mid \theta\left(t_{k}\right)\right]=H\left(t_{k}\right)$ independent of $0\left(t_{k}\right)$, which gives particularly simple results.

At $t=t_{3}$, we have

$$
p\left[Z_{1} \mid \theta\left(t_{1}\right), \theta\left(t_{0}\right), \sigma\left(t_{1}\right)\right]=\left\{2 \pi \alpha\left(t_{1}\right)\right\}^{-\frac{3}{2}}\left\{1-H\left(t_{1}\right) k\left[t_{1} \mid \theta\left(t_{1}\right)\right]\right\}^{\frac{2}{2}} \exp \left\{-\frac{e\left[t_{1} \mid \theta\left(t_{1}\right)\right]}{2 \alpha\left(t_{1}\right)}\right\}
$$

where

$$
\begin{aligned}
& \dot{x}\left[t_{1} \mid \theta\left(t_{1}\right)\right]=\left\{1-H\left(t_{1}\right) K\left[t_{1} \mid \theta\left(t_{1}\right)\right]\right\} \tilde{z}^{2}\left[t_{1} \mid \theta\left(t_{0}\right)\right] \\
& \hat{z}\left[t_{1} \mid \theta\left(t_{0}\right)\right]=z\left(t_{1}\right)-H\left(t_{1}\right) \hat{x}\left[t_{1} \mid t_{0}, \theta\left(t_{0}\right)\right]
\end{aligned}
$$

Observe that, for each $\left[\theta\left(t_{1}\right), \theta\left(t_{0}\right)\right], p=p\left[z_{1} \mid \theta\left(t_{1}\right), \theta\left(t_{0}\right), \alpha\left(t_{1}\right)\right]$ is a unimodel function of $a\left(t_{1}\right)$. Setting $\frac{\partial p}{\partial \alpha\left(t_{1}\right)}=0$ gives $\hat{\alpha}\left(t_{1}\right)=\alpha\left[t_{1} \mid O\left(t_{1}\right)\right]$ and $p\left[z_{1} \mid \theta\left(t_{1}\right), \theta\left(t_{0}\right), \hat{\alpha}\left(t_{1}\right)\right]=c\left|\tilde{z}\left[t_{1} \mid \theta\left(t_{0}\right)\right]\right|$. Choosing $\hat{\theta}\left(t_{0}\right)$ to maximize $\left\{\mid \tilde{z}\left[t_{1} \mid \theta\left(t_{0}\right)\right]\right\}$, the one step ahead prediction state estimate given by the iv Kalman filters are then reset to $\hat{x}\left[t_{1} \mid t_{0}, \theta\left(t_{0}\right)\right]$. (Note that in this case $\hat{\theta}\left(t_{0}\right)$ is independent of $\theta\left(t_{1}\right)$ so that the calculations are much simplified.)

By induction, we easily obtain for each $\left[\theta\left(t_{k}\right), \theta\left(t_{k-1}\right)\right]$

$$
\hat{\alpha}\left(t_{k}\right)=x\left[t_{k} \mid \theta\left(t_{k}\right), \theta\left(t_{k-1}\right), \theta\left(t_{k-2}\right)\right]
$$




$$
p\left[Z_{k} \mid \theta\left(t_{k}\right), \theta\left(t_{k-1}\right), \hat{O}\left(t_{k-2}\right), \hat{A}\left(t_{k}\right)\right]=c^{\prime}\left|z\left[t_{k} \mid \cdots\left(t_{k-1}\right),\left(t_{k-2}\right)\right]\right|
$$

where $\left.x\left[t_{k} \mid \theta\left(t_{k}\right), \theta\left(t_{k-1}\right), \hat{O}\left(t_{k-2}\right)\right]=\left\{1-H\left(t_{k}\right) K\left[t_{k} \mid \cdots\left(t_{k}\right)\right]\right\} z^{2}\left[t_{k}\right) \cdot\left(t_{k-1}\right),\left(t_{k-2}\right)\right]$ $\tilde{z}\left[t_{k} \mid \theta\left(t_{k-1}\right), \hat{0}\left(t_{k-2}\right)\right]=z\left(t_{k}\right)-H\left(t_{k}\right) \hat{x}\left[t_{k} \mid t_{k-1}, \because\left(t_{k-1}\right), \hat{i}\left(t_{k-2}\right)\right]$. The normalising constant $c^{\prime}$ and the sequence $\hat{c}\left(t_{k-2}\right)$ are independent of $\left[0\left(t_{k}\right), 0\left(t_{k-1}\right)\right]$.

From equation (34), $p\left[z_{k} \mid \theta\left(t_{k}\right), \theta\left(t_{k-1}\right),\left(t_{k-2}\right), A\left(t_{k}\right)\right]$ is independent of $\theta\left(t_{k}\right)$. Thus, the problem of finding the global maximum of $p\left[z_{k} \mid \theta\left(t_{k}\right), \theta\left(t_{k-1}\right), \hat{O}\left(t_{k-2}\right), \alpha\left(t_{k}\right), \hat{A}\left(t_{k-1}\right)\right]$ with respect to $\left[\cdot\left(t_{k-1}\right),\left(t_{k}\right)\right]$ reduces to the problem of finding $\min \left\{z^{2}\left[t_{k} \mid{ }_{i}\left(t_{k-1}\right),\left(t_{k-2}\right)\right]:\right.$ The one step ahead prediction state estimate given by the li Kalman filters are then reset to $\hat{x}\left[t_{k} \mid t_{k-1}, \hat{\ominus}\left(t_{k-1}\right)\right]$. The estimator structure for this problem is illustrated diagramaticaliy in figure 4.

\section{Example}

A modification of the first-order system aiven by equations (25) and (26) is used to compare the performances of the estimators described in the last section and the estimator described in this section. Here we assume $G, J$ to be uniformily distributed white noise sequences. Figure 5 shows a typical set of simulation results which demonstrate that the estimator described in this section gives superior performance when the unknown parameters are varying rapidly with time.

It might be thought that the scheme proposed in this section could be better than that of the previous section for the example of the previous section since the unknown parameter is really time-varying al though 5 lowly varying in this example. Simulations showed that when the parameter variations are slowly varying the non-resetting scheme of the previous section works best. 
IV CONTIMUOUS-TIME RESULTS.

The'maximum likelihood' technique clescribed in this paper has the advantage that even for continuous-time measurements, an estimator can be obtained which does not require the exact knowledge of the measurement noise covariance.

Time-invariant unknown paraneters

Consider the system

$$
\begin{aligned}
& \dot{x}(t)=F(t)) x(t)+G(t \mid) u(t) \quad t=0 \\
& y(t)=H(t) j x(t) \\
& z(t)=y(t)+v(t)
\end{aligned}
$$

where $\{u(t)\},\{(t)\}$ are independent zero mean Gaussian white noise processes with covariance matrices $I, P(t)$ respectively. The initial state $x\left(t_{0}\right)$ is a Gaussian random vector with mean $\hat{x}\left(t_{0} \mid t_{0}, \dot{w}\right)$ and variance $P\left(t_{0}, t_{0}, t\right)$ and is indeperident of $\{u(t)\}$ and $\{v(t)\}$. The measurement noise covariance $R(t)$ is assuned to be of the form $R(t)=a R_{0}(t)$ where $R_{i}(t)$ is known but is is an unknown scalar. The entities $F(t \mid w), G(t \mid \psi), H\left(\iota \mid \psi j, \therefore\left(t_{0} \mid t_{0}, \psi\right), P\left(t_{0} \mid t_{0}, t\right)\right.$ are known if the parameter vector $\psi$ is known, possibly unknown if is is not known.

If 4 and a are specified, the conditional mean state estimate $\hat{x}(t \mid t, \psi, x)$ is given from the kalman-Bucy filter equations:

$$
\dot{x}(t \mid t, \psi, \alpha)=F(t \mid \psi) \hat{x}(t \mid t, \psi, \alpha)+k(t \mid \psi, \alpha)[z(t)-\hat{y}(t \mid t, \psi, \alpha)]
$$




$$
\begin{aligned}
& \hat{y}(t \mid t, \psi, \alpha)=H(t \mid \psi) x(t \mid t, \psi, i t) \\
& K(t \mid \psi, \alpha)=P(t \mid t, \psi, i) H^{-}(t \mid+)\left[R_{0}(t)\right]^{-} \\
& \dot{P}(t \mid t, \psi, \alpha)=F(t \mid \psi) P\left(t \mid t, w_{1}\right)+P(t \mid t, \omega) F^{\prime}(t \mid b)-K(t \mid,, 1) \\
& R_{0}(t) K^{-}(t \mid \psi, w)+G(t \mid,) G^{-}(t \mid)
\end{aligned}
$$

If $\alpha$ is specified but $\psi$ is unknown, then assuming $i$, the space of admissible values of $\psi$, is discrete or suitably guantized to a finite number of grid points $\left\{\psi_{1}, \ldots, \psi_{p}\right\}$ with known or assumed a priori probability $p\left(\psi_{j}, t, \alpha\right)$ for each $\psi_{j}$, the conditional mean state estimate $\hat{x}(t \mid t, a)$ is given by [5]:

$$
\begin{aligned}
& \hat{x}(t \mid t, u)=\sum_{j=1}^{p} \hat{x}\left(t \mid t, \psi_{i}, \alpha\right) p\left(\psi_{i} \mid t, \ldots\right) \\
& p\left(\psi_{j} \mid t, a\right)=c \exp \left(l\left(\psi_{j} \mid t, r\right)\right) p\left(\psi_{j} \mid t_{0}, \lambda\right)
\end{aligned}
$$

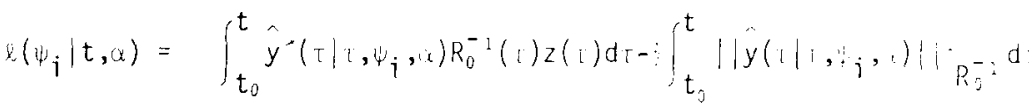

where $c$ is a normalizing constant independent of $i j$ and $(j \mid t, i)$ is the $\log$ likelihood function of $i$ given and the measurements $\left\{z(\tau)\left\{\tau \in\left(t_{0}, t\right)\right\}\right.$.

Again, allowing for more parallel processing effort, it might appear to the reader that a natural extension of the above results for the case when $\alpha$ is unspecified is 'readily achieved' using the following approach. Assume A, the range of admissible values of $\alpha$, can be approximated by the finite set $\left\{\alpha_{1}, \ldots, \alpha_{M}\right\}$. Then, with known or assumed a priori probability 
$p\left(\psi_{i}, x_{j} \mid t_{u}\right)$ for each $\left(\psi_{i}, x_{j}\right)$, the conditional mean state estimate would be given from

$$
x(t \mid t)=\sum_{j=1}^{P} \sum_{j=1}^{M} \hat{x}\left(t \mid t, \psi_{j}, w_{j}\right) p\left(\psi_{j}, x_{j} \mid t\right)
$$

However, further difficulty arises because there is no direct and simple technique to recursively update $p\left(\psi_{i}, u_{j} \mid t\right)$.

We now propose a simple estimation scheme to yield a suboptimal minimum mean square error estimate for the case when a is unknown. Following the approach described in section II we shall now consider directly the space ;) of hypotheses of possible kalman filter configurations. For each $\theta$ in $\because$, the conditional mean state estimate $\hat{x}(t \mid t, \theta)$ is given by the following equations:

$$
\begin{aligned}
& \dot{x}(t \mid t, 0)=F(t \mid 0) \hat{x}(t \mid t, \theta)+k(t \mid 0)[z(t)-\hat{y}(t \mid t, 0)] \\
& \hat{y}(t \mid t, \theta)=H(t \mid \theta) \hat{x}(t \mid t, 0)
\end{aligned}
$$

Again, we approximate 0 with a finite set of grid points $\left\{0_{1}, \ldots, 0_{N}\right\}$, and we make the simplifying assumption that each "i allows the same range of admissible values of $a$. Then, for any admissible value of $\alpha$, the log likelihood function $\left.x_{i} \mid t, \alpha\right)$ of $\theta_{j}$ given the measurements $\left\{z(\tau) \mid \tau \in\left(t_{0}, t\right)\right\}$ can be expressed as

$$
\ell\left(\theta_{i} \mid t, \alpha\right)=L\left(\theta_{i} \mid t\right) / \alpha
$$


where

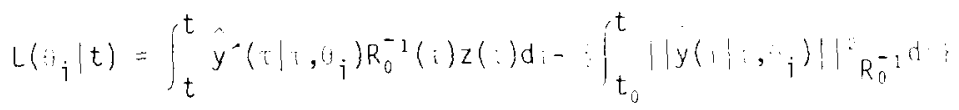

Obviously, given the measurements $\left\{z(1) \mid: \epsilon\left(t_{0}, t\right) !\right.$, the most likely ${ }_{i}$, denoted $\hat{\theta}$, is the $\theta_{i}$ which maximizes $L\left(a_{i} t\right)$, which is independent of $i x$. This observation suggests that in the event that " is not known, a very simple state estimator is one yielding the estimate $\hat{x}(t \mid t, i)$. Such an estimator is sketched diagramatically in Figure 5. Simulation results have not been obtained for this estimator since it is not anticipated that they can extablish anv more than those for the riscrete-time results.

\section{Time-Varying Unknown Parameters}

Here we consider obtaining estimators when the unknown parameter vector $\theta(t)$ and the measurement noise intensity a are modelled as piecewise constant functions of time with $\theta(t)=\theta\left(t_{k}\right), \omega_{a}(t)=\left(t_{k}\right)$ for $t_{k} \leq t<t_{k+1}$. We further assume that $\theta\left(t_{k}\right)$ can take one of the values $\left\{\theta_{1}, \ldots, \hat{\theta}_{N}\right\}$.

The estimation scheme that we propose is as follows.

At $t=t_{1}, \hat{\theta}\left(t_{0}\right)$ is found such that $L\left[\hat{\theta}\left(t_{0}\right) \mid t_{1}\right]=\max _{i}\left[t_{i}\left(t_{0}\right) \mid t_{1}\right]$. The states of all the $N$ Kalman filters are then reset to $x\left[t_{1} \mid t_{1}, i\left(t_{0}\right)\right]$, viz, the state of the Kalman filter conditioned on $\left(t_{0}\right)$. In general, at

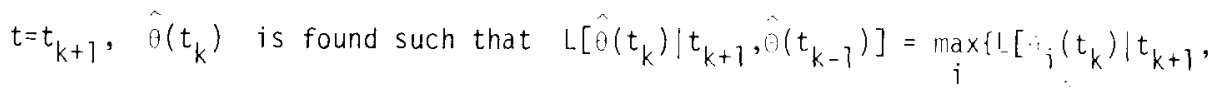
where $\hat{\theta}\left(t_{k-1}\right)=\left\{\hat{\theta}\left(t_{0}\right), \ldots, \hat{\theta}\left(t_{k-1}\right)\right\}$ and $L\left[\theta_{i}\left(t_{k}\right) \mid t, \hat{\theta}\left(t_{k-1}\right)\right]=\int_{t_{k}}^{t} \hat{y}^{-}\left[\tau \mid \tau, \hat{O}\left(t_{k-1}\right), \theta\left(t_{k}\right)\right] R_{0}^{-1}(\tau) z(\tau) d \tau-\int_{t_{k}}^{t}|| \hat{y}(i \mid i$, $\left.\hat{O}\left(t_{k-1}\right), \theta\left(t_{k}\right)\right)||^{2} R_{0}^{-} d:$ 
Simulations have not been carried out for this scheme since as for the previous not too much additional information can be gained from this. However, it could be stressed that the important point here is that the ideas investigated in this paper, in contrast to the standard Bayesian approach, can in fact be applied to continuous-time systems in a straightforward manner. 
[1] R.E. Kalman, "A New Approach to Linear Filtering and Prediction Problems", Trans. ASME, J. Basic Engng., Vol. 82, March 1960, pp. $34-45$.

[2] R.E. Kalman and R.S. Bucy, "New Results in Linear Filtering and Prediction Theory", Trans. ASME, J. Basic Engng., sec. D Vol. 83, December 1961, pp. 95-107.

[3] D.T. Magi1], "Optimal Adaptive Estimation of Sam led Stochastic Processes" IEEE Trans. Automat. Contr., Vo1. AC-10. No 4 Oct 1965, pp. $215-218$.

[4] F.L. Sims, D.G. Lainiotis and D.T. Magi71, "Recursive Algorithmi for the Calculation of the Adaptive Kalman Filter weighting coefficients," IEEE Trans. Automat. Contr. (Corresp.), Vol. AC-14, April 1969.

[5] D.G. Lainiotis, "Optimal Adaptive Estimation: Structure and Parameter Adaptation", IEEE Trans. Automat. Contr. Vol. AC-16, No. 2, April 1971 pp. 160-169.

[6] D.L. Alspach and A. Abiri, "Optimal Nonlinear Estimation for a Linear System with Unknown Plant and Measurement Noise Covariances", Proc. of 3rd Symposium on Nonlinear Estimation Theory and its Applications, San Diego, September 1972, pp. 11-13.

[7] D.L. Alspach, "A Parallel Processing Solution to the Adaptive Kalman Filtering Problem with Vector Measurements", Intern. J. Computers and Electrical Engng., Vol. I No. 1, June 1973, pp 83-94.

[8] D.L. Alspach, "A Bayesian Nonlinear Filtering Algorithm for Linear Systems with Unknown Time Varying Noise Statistics", Proc. IEEE Conference on Decision and Control incl. 12th Symposium on Adaptive Processes, December 1973, pp. 533-539.

[9] C.G. Hilborn, Jr., and D.G. Lainiotis, "Optimal Adaptive Filter Realizations for Sample Stochastic Processes with an unknown Parameter" IEEE Trans. On Automat. Contr. Vol AC-14, December 1969, pp. 767-770.

[10] G.A. Ackerson and K.S. Fu, "On State Estimation in Switching Environments" IEEE Trans. Automat. Contr. Vol AC-15, February 1970, pp. 10-16. 


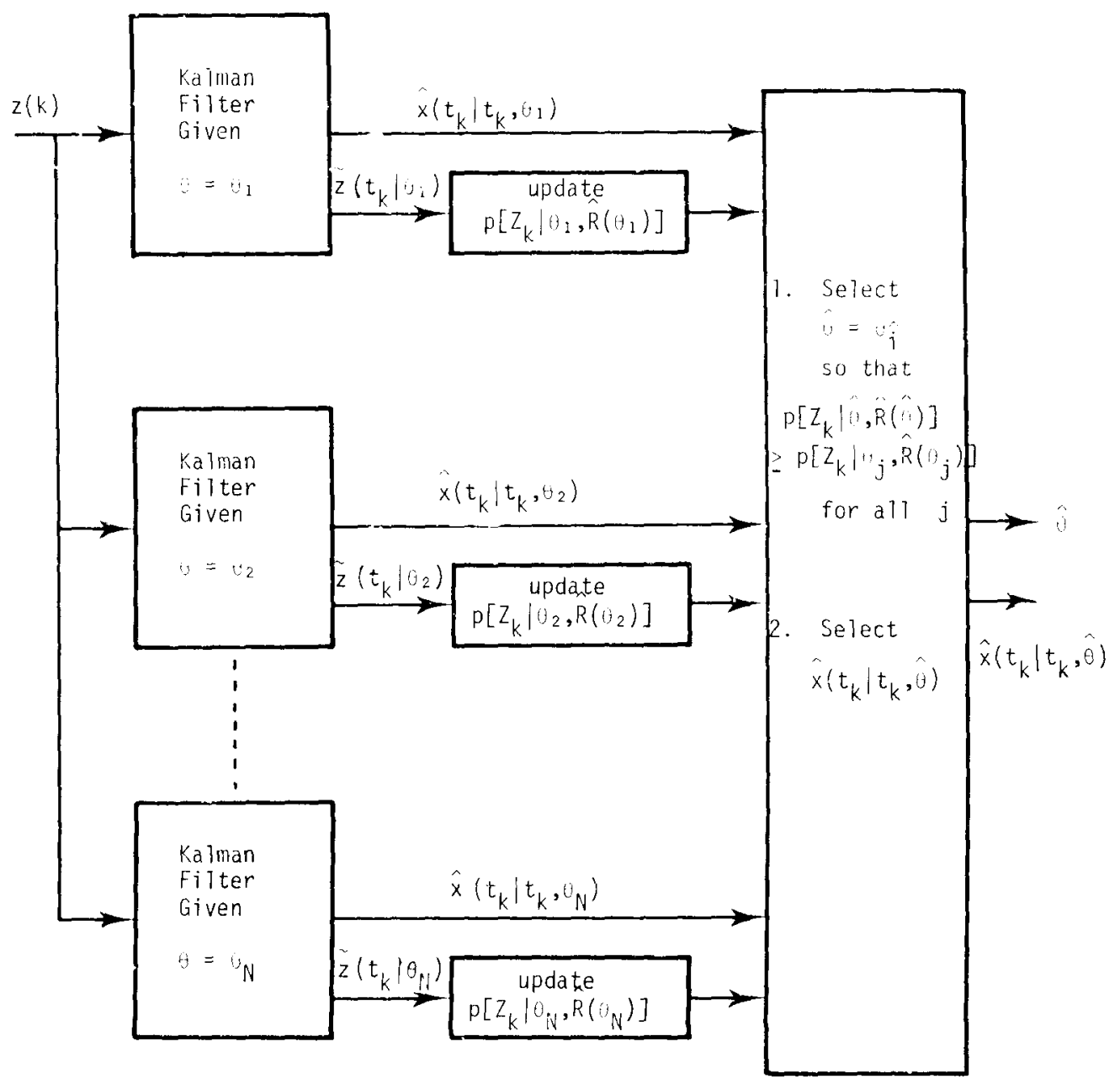

FIG. 1 DISCRETE-TIME ADAPTIVE ESTIMATOR FOR TIME-INVARIANT $\theta$. 


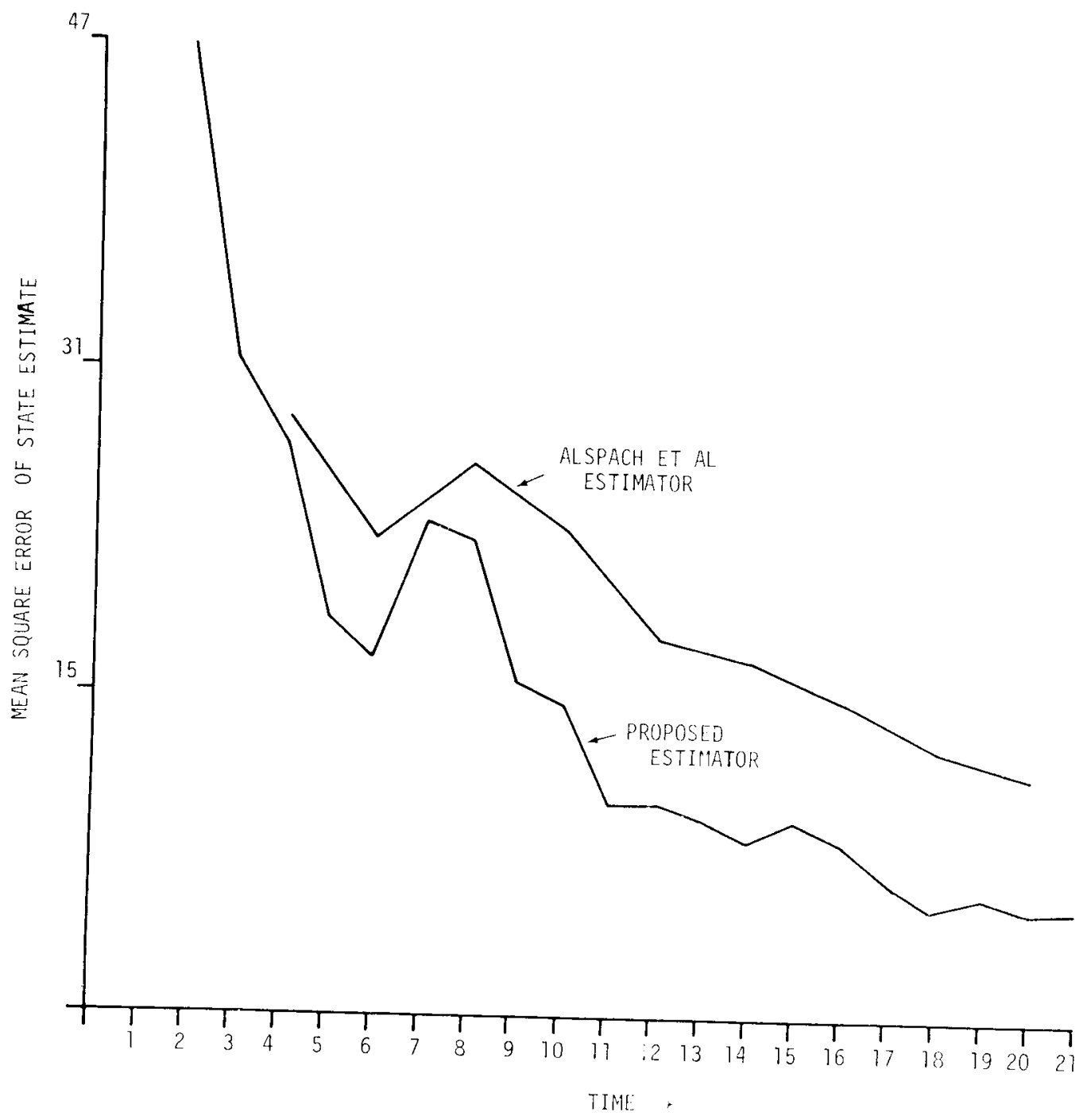

Fig. 2 MEAN SQUARE ERROR FOR STATE ESTIMATION IN HIGH NOISE $(G=.6, \mathrm{~J}=10)$ 


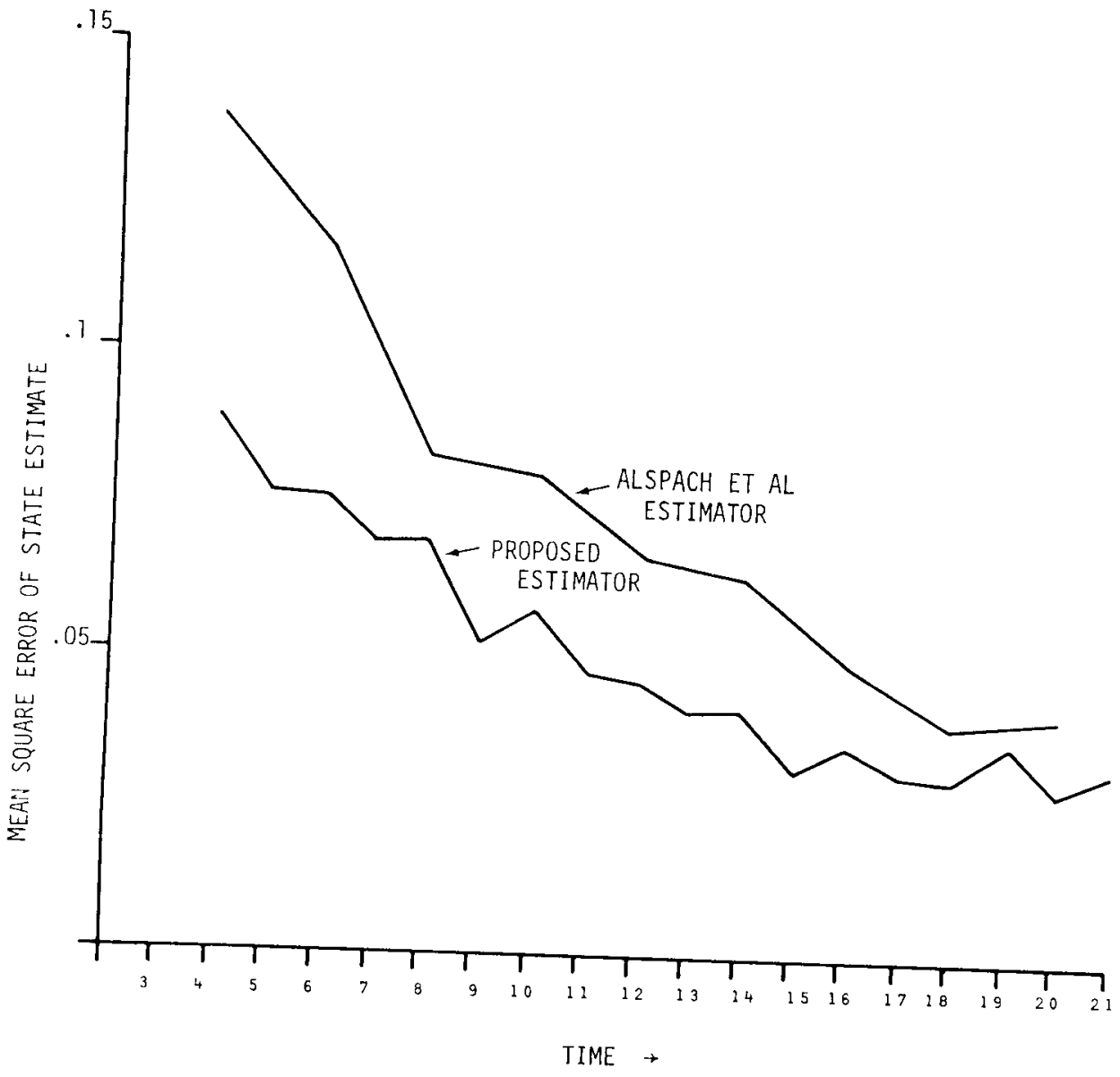

FIG. 3 MEAN SQUARE ERROR FOR STATE ESTIMATION IN LOW NOISE $(G=.6, \mathrm{~J}=.1$ ) 


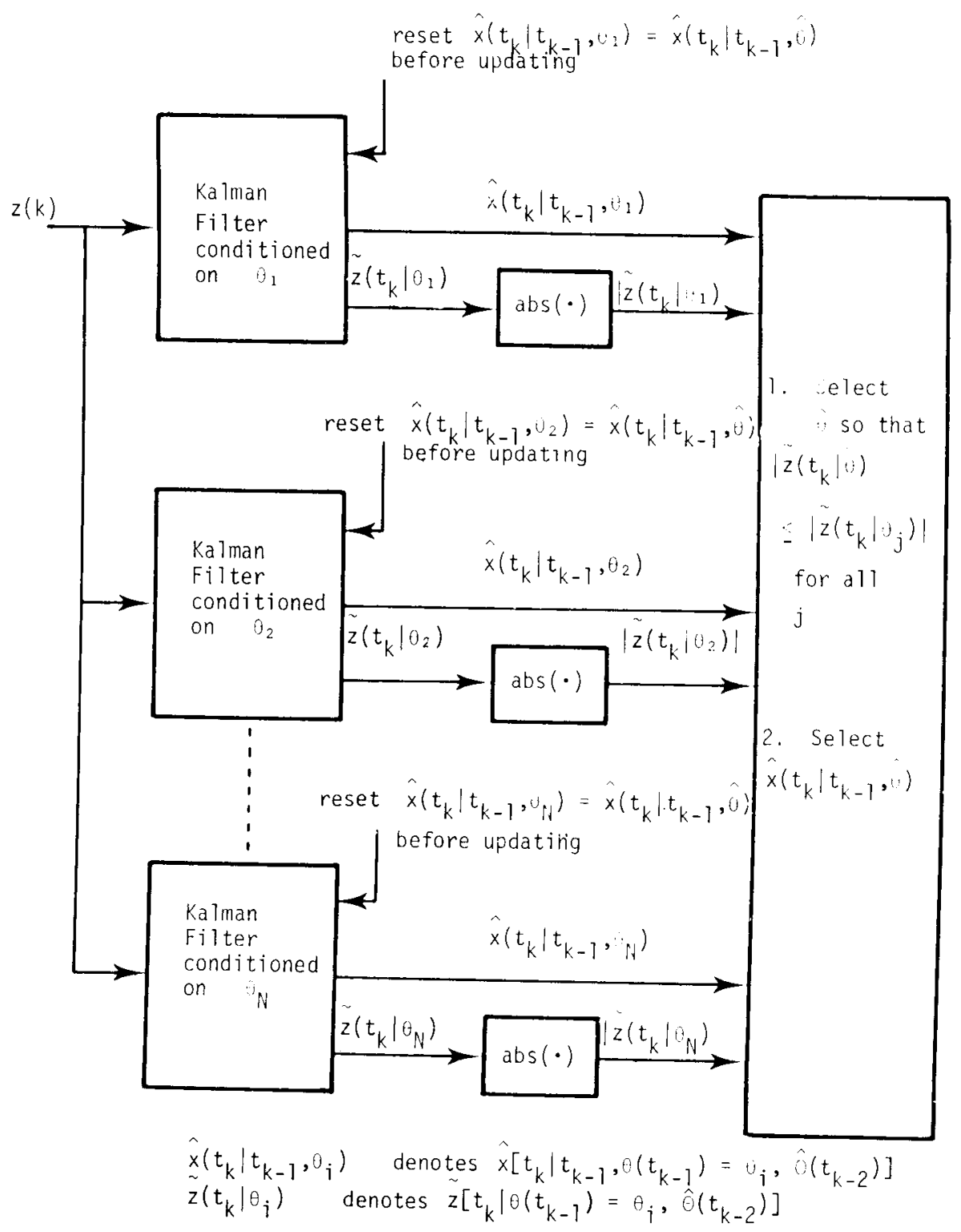

FIG. 4 DISCRETE-TIME ADAFTIVE ESTIMATOR FOR TIME-VARYING [ASSUMING SCALAR MEASUREMENTS AND $\left.H\left[t_{k} \mid \theta\left(t_{k}\right)\right]=H\left(t_{k}\right)\right]$ 


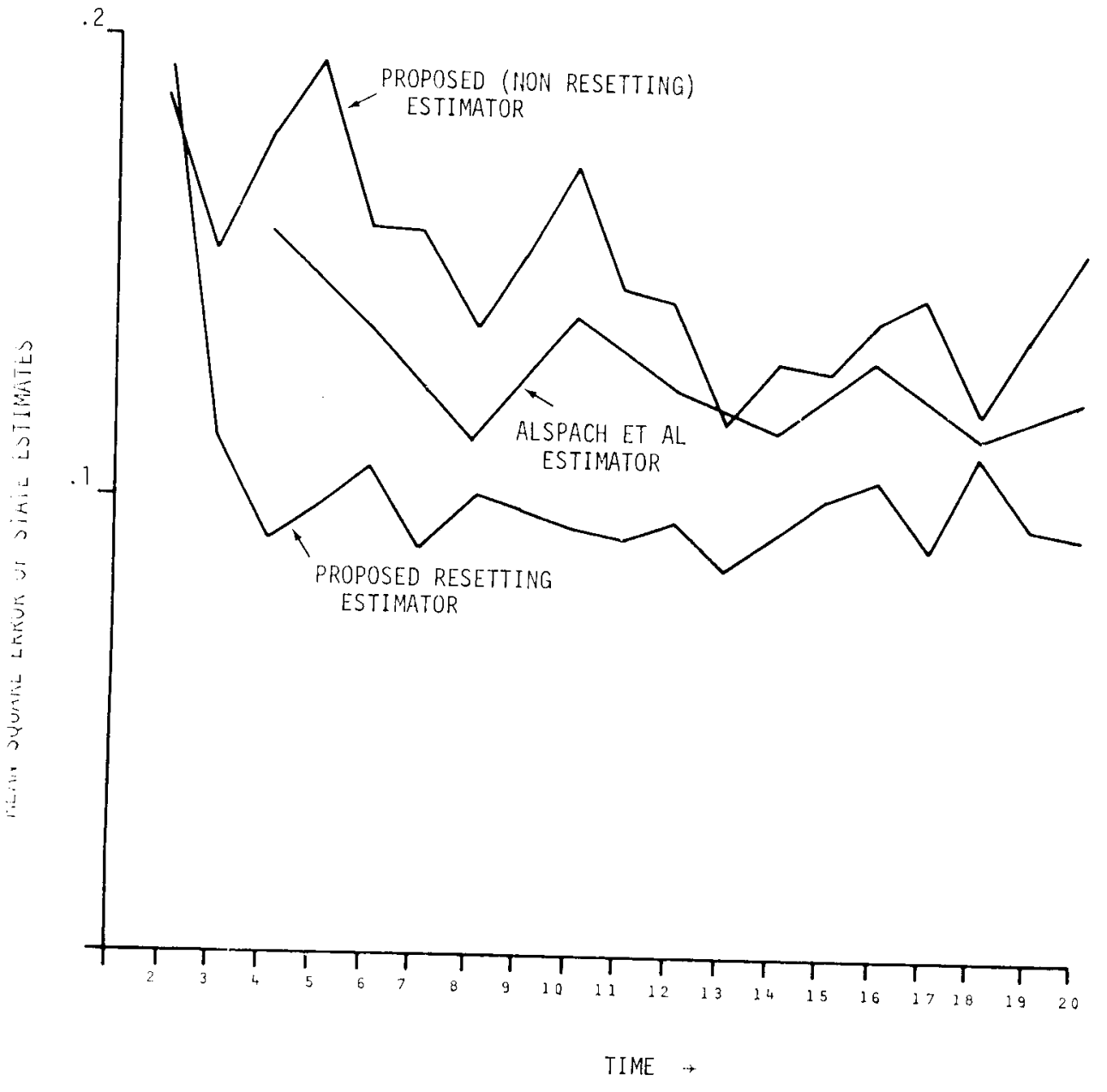

FIG. 5 MEAN SQUARE ERROR OF STATE ESTIMATES FOR TIME-VARYING NOISE COVARIANCES $[\hat{\alpha}, J$ RANDOM VARIABLES UNIFORMLY DISTRIBUTED IN $(0,1)]$ 


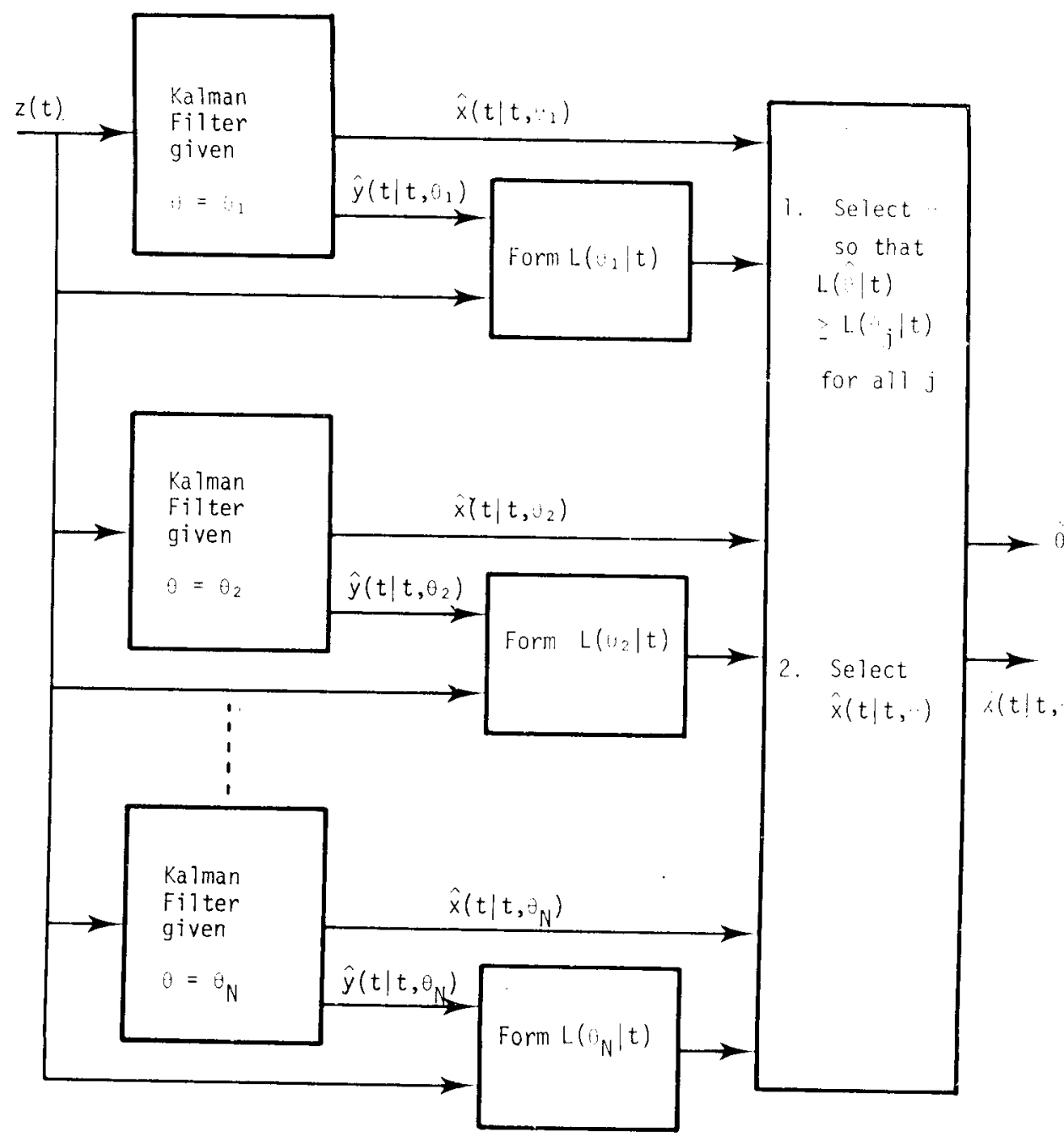

FIG. 6 CONTINUOUS-TIME ADAPTIVE ESTIMATOR FOR TIME-INVARIANT 


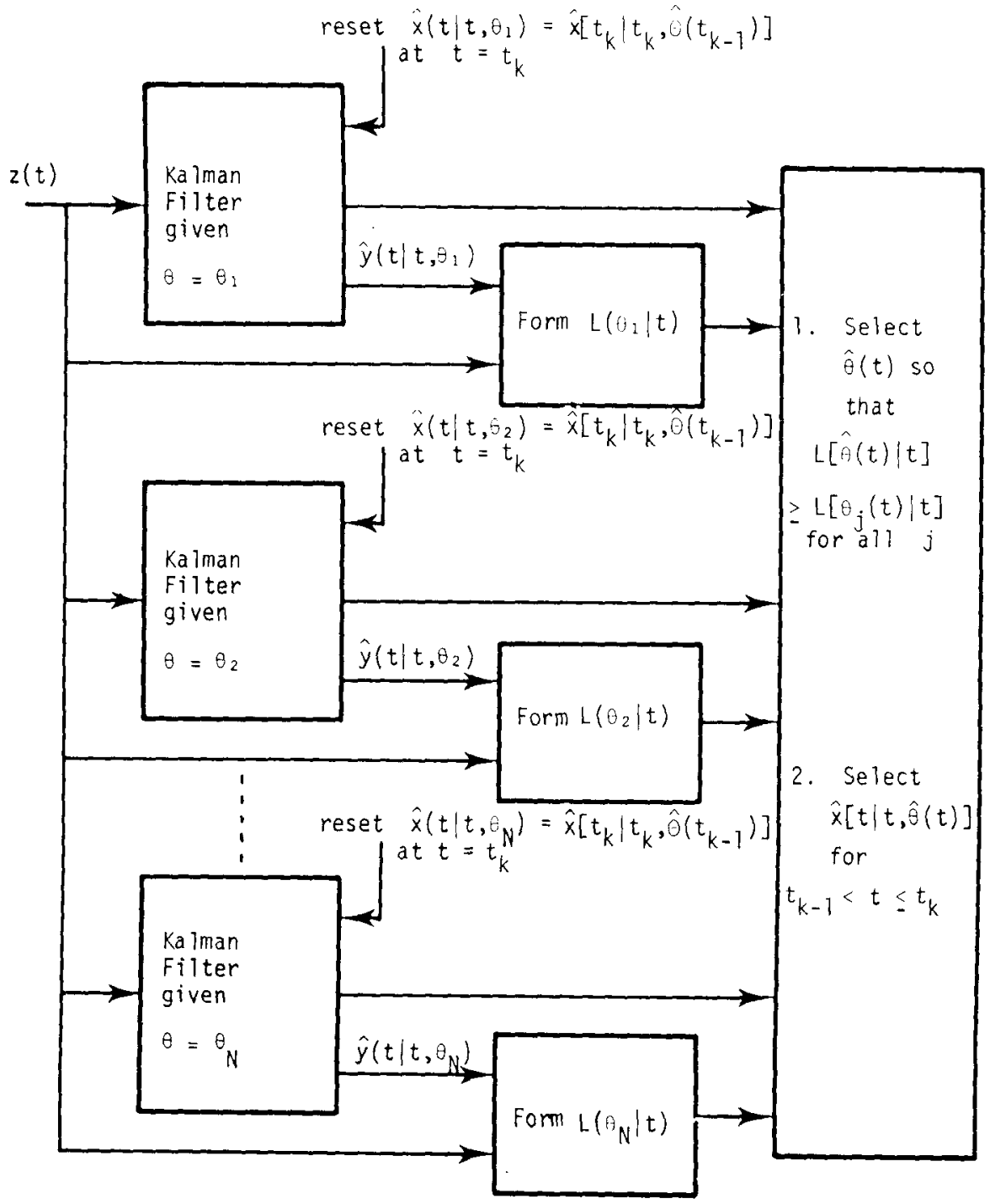

FIG. 7 CONTINUOUS-TIME ADAPTIVE ESTIMATOR FOR TIME-VARYING $\theta$ 